\title{
Elastic Stress Relaxation in HRTEM Specimens of Strained Semiconductor Heterostructures and its Influence on the Image Contrast
}

\author{
Liberato De $\operatorname{Caro}\left({ }^{1}\right)$, Antonino Giuffrida $\left({ }^{2}\right)$, Elvio $\operatorname{Carlino}\left({ }^{1}\right)$ and Leander $\operatorname{Tapfer}\left({ }^{1}\right)$ \\ ( $\left.{ }^{1}\right)$ PASTIS-Centro Nazionale Ricerca e Sviluppo Materiali (PASTIS-CNRSM), Strada Statale 7 \\ Appia km 712, 72100 Brindisi, Italy \\ $\left({ }^{2}\right)$ Università degli Studi di Lecce, Dipartimento di Fisica, via Arnesano, 73100 Lecce, Italy
}

(Received March 1; accepted May 15, 1995)

\begin{abstract}
HRTEM observations require ultra-thinned specimens and, due to the very small thickness ( 1 to $30 \mathrm{~nm}$ ), an elastic stress relaxation may occur near the free surfaces of strained heterostructures. These relaxation phenomena can modify the structural features of the thinned specimens with respect to the bulk materials. Thus, the role of the relaxation has to be taken into account if the structural properties of the bulk heterostructures are deduced from those of the thinned specimens. In this work we investigate the case of lattice-mismatched semiconductor superlattices, thinned along the [011]-crystallographic direction. The theoretical evaluation of strain fields in ultra-thinned HRTEM samples shows a bending of the lattice, and the local lattice spacings are representative of neither the bulk tetragonally distorted material nor the unstressed material. Our results show that these distortions can be large and must be taken into account whenever HRTEM is used to deduce the local chemical composition or the unit cell dimensions in strained semiconductor materials.
\end{abstract}

\section{Introduction}

In order to exploit the novel electronic and opto-electronic properties of strained semiconductor heterostructures, an accurate knowledge about the chemical composition, the structural parameters and the strain distribution is a fundamental prerequisite. In particular, the structural status of the heterointerfaces plays an important role on the physical properties of this class of materials. In order to achieve local structural information, transmission electron microscopy (TEM) techniques like high-resolution TEM (HRTEM) are widely used.

As HRTEM observations require ultra-thinned specimens (1 to $30 \mathrm{~nm}$ thick), elastic stress relaxation phenomena can considerably modify the lattice deformations of the thinned heterostructures. In several works attempts have been made to measure superlattice misfit strains from selected area diffraction spot splitting and high resolution lattice imaging of interfaces in crosssection $[1,2]$. Since these workers neglected surface relaxation effects, their results are representative of the strain status of the thinned specimen but not of the bulk heterostructure. 
In order to overcome this problem Treacy et al. [3-5] and Perovic et al. [6] studied surface relaxation effects in strained heterostructures, thinned along the [100]-crystallographic direction, by means of a Fourier-series elasticity treatment. These authors focused their attention on the influence of the elastic relaxation in the diffraction contrast imaging. They showed that, due to relaxation of compositional stresses, the lattice plane bending near the specimen surfaces can cause strong diffraction contrasts in TEM bright-field and dark-field images. They argued that even the interpretation of high-resolution lattice images should be affected by stress relaxation near the free surfaces.

One attempts in this direction was made in the work of reference [7] where, assuming a sinusoidal lattice bending in a GaAs crystal, it was shown that an evident contrast in HRTEM simulated images can occur. However, the authors of this work did not calculate the lattice bending in the framework of the elasticity theory, but simply they assumed a sinusoidal strain distribution with an amplitude proportional to the InAs-GaAs lattice mismatch.

Up to now no rigorous and systematic study on this subject has been reported. The aim of our study is to demonstrate the influence of the elastic relaxation, calculated within the framework of the elasticity theory, on the HREM image contrast. Since most of the semiconductor heterostructures analyzed in cross-section are thinned along the [011]-crystallographic direction, in our Fourier-series elasticity treatment we have assumed this particular thinning geometry. The relevant calculations will be reported elsewhere $[8,9]$. In Section 2 we summarize the main results. In particular it is shown that the lattice deformation is non-uniform in all the superlattice period and for all the thickness of the specimen. The non-uniformity of the lattice deformation implies that it is necessary to calculate the position of thousands of atoms in order to create the supercell necessary for HRTEM simulations. Using the software MATHEMATICA, [10] running on a workstation RISC 6000 mod. 560, we implemented a computer program which allows us to create these extended supercelles (see Sect. 3). In particular, the implemented computer program is compatible with the simulation package CERIUS-HRTEM, [11] running on a workstation RISC 6000 mod. 350 equipped with a graphic card SABINE 24 bit. In fact, all the parameters of the supercell (type of atom, atomic coordinates, etc.) are given in the format required by this simulation program. For particular defocus values our simulations show an evident contrast variation due to the non-uniform lattice deformation predicted by the elasticity theory.

\section{Calculation of the Elastic Lattice Deformation in HREM Specimens of Strained Semiconduc- tor Superlattices}

Let us consider a strained superlattice of period $T$, made of two cubic semiconductor materials A and B with thicknesses $a$ and $b$, and lattice parameters $d_{a}$ and $d_{b}$, respectively. Let us suppose that the superlattice has been grown along the $x$ crystallographic direction and that it has been thinned along the $z$ direction, for the HRTEM specimen preparation, with final thickness $t$, (Fig. 1). Moreover, let us assume that along the other orthogonal direction $y$ the dimension of the superlattice is infinite. Now, we need to calculate the strain and the stress fields induced by the difference of lattice parameters of the two constituent materials A and B, and modified by the partial elastic relaxation of the compositional stresses on the free surfaces after the thinning process. In particular, we are interested to the case in which $x=[100], y=[0 \overline{1} 1] / \sqrt{2}$ and $z=[011] / \sqrt{2}$.

Stress calculations for strained superlattices thinned along the [011]-crystallographic direction are analogous to those made by Treacy and Gibson [3, 5], which assumed the [001] thinning direction. In fact, even in this case we are dealing with a plane deformation problem $\left(\varepsilon_{y y}^{\mathrm{el}}=0\right)$ due to the assumed infinite dimension of the superlattice along the $y$ direction (see Fig. 1). For this reason, all the stress and strain components are functions of the $x$ and $z$ coordinates. The only 


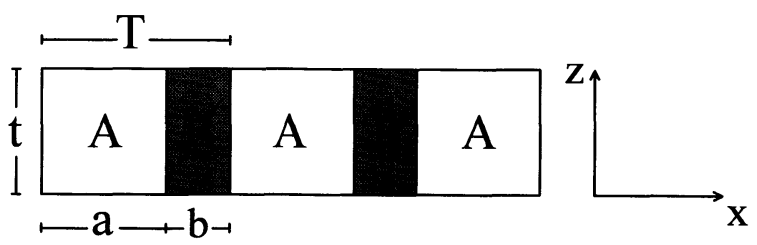

Fig. 1. - Schematic view of a thinned strained superlattice of period $T$ and thickness $t$, made of two cubic semiconductor materials $\mathrm{A}$ and $\mathrm{B}$ with thicknesses $a$ and $b$ and lattice parameters $d_{a}$ and $d_{b}$, respectively.

difference is related to the fact that in this case the involved strain components are not coincident with those calculated with respect to the crystallographic axes [100], [010] and [001], but with respect the reference system shown in Figure 1.

By using the Hooke's equations, the strain compatibility conditions and the equations of equilibrium in absence of body forces [12], we obtain the following differential equation:

$$
m \frac{\partial^{2} \sigma_{x x}^{\mathrm{el}}}{\partial z^{2}}+\frac{\partial^{2} \sigma_{z z}^{\mathrm{el}}}{\partial x^{2}}-n \frac{\partial^{2} \sigma_{x z}^{\mathrm{el}}}{\partial x \partial z}=0
$$

where

$$
\begin{aligned}
& n=\frac{\alpha(1+\nu)+1-3 \nu}{1-\nu} \\
& m=\frac{1+\alpha-2 \nu}{2 \alpha(1-\nu)}
\end{aligned}
$$

Here $\alpha=\frac{C_{11}-C_{12}}{2 C_{44}}$ is the anisotropy coefficient, $\nu=\frac{C_{12}}{C_{11}+C_{12}}$ is the Poisson ratio, $C_{i j}$ are the elastic stiffness constants which are assumed to be equal in the two cubic semiconductor materials $\mathrm{A}$ and $\mathrm{B}$. In equation (1) only the elastic contribution to the stress and strain tensor components appears. In fact, the compositional contribution, due to the lattice mismatch between the lattice parameters of the two materials, can be taken into account by means of the strain suppression method [3,5]. It should be noted that the coefficients $m$ and $n$ of the differential equation (1) differ with respect to those obtained for superlattices thinned along the [100] crystallographic direction $[3,5]$.

We can calculate the stress field in the $(x, z)$ plane of the superlattice by defining the stress components as a function of the stress function [3,5], expanding in a Fourier series the latticemismatch $\varepsilon(x)$ of the periodic strained superlattice and imposing the boundary conditions of zero total stress components on the free surfaces for each $m$-th Fourier wave [8,9]. At this point, by using Hooke's law, we can obtain the strain components $[8,9]$.

In Figure 2 we show the total atomic displacement field in the $(x, z)$ plane for a Si/Ge superlattice with period $T=4.91 \mathrm{~nm}, a=4.34 \mathrm{~nm}, b=0.57 \mathrm{~nm}$, and thickness $t=5 \mathrm{~nm}$. It is interesting to note that near the free surfaces a bending of the lattice occurs. The lattice distortion is present in both the germanium and silicon layers and causes a contrast in the HREM images, independently from the chemical contrast due to the different atomic species, as it will be shown in the next section. 


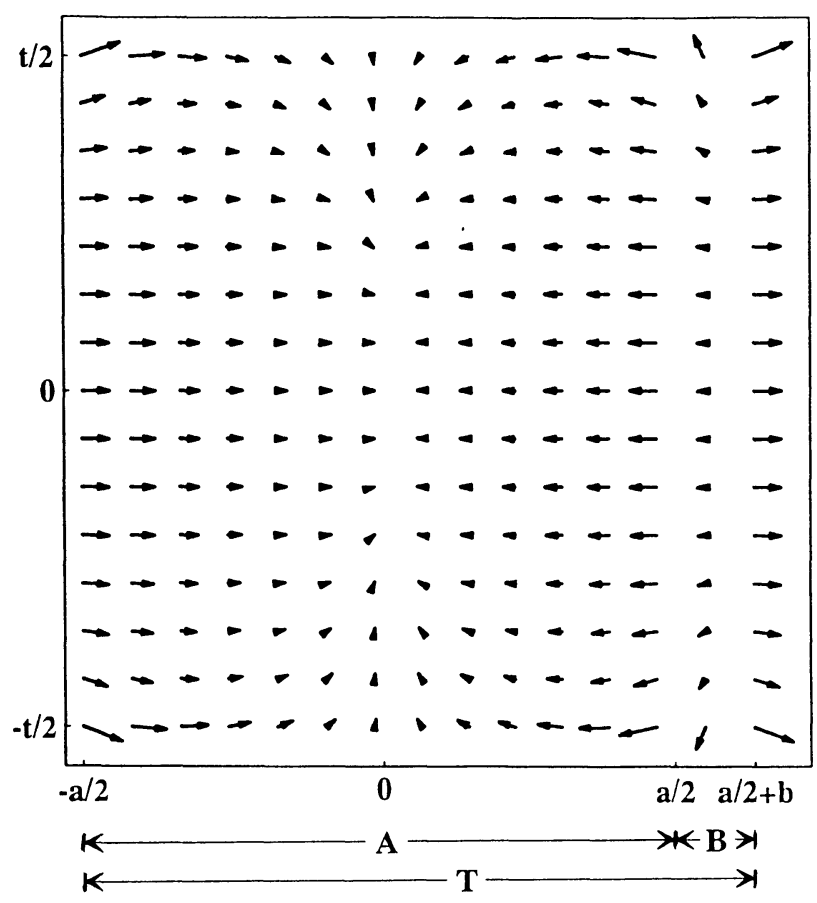

Fig. 2. - Total atomic displacement field in the $(x, z)$ plane for a Si/Ge superlattice with period $T=$ $4.91 \mathrm{~nm}, a_{\mathrm{Si}}=4.34 \mathrm{~nm}, b_{\mathrm{Ge}}=0.57 \mathrm{~nm}$, and thickness $t=5 \mathrm{~nm}$. The arrows are the atomic displacement vectors, with respect to the undeformed positions, magnified by a factor 2 .

\section{Influence of the Elastic Lattice Relaxation in Strained Semiconductor Superlattices on the HREM Image Contrast}

Figure 2 shows a non-uniform lattice deformation everywhere in the Si/Ge superlattice period. Along the $z$-axis the atom positions lost their translational symmetry. Along the $x$-axis the superlattice periodicity remains unchanged. Only along the $y$-axis the lattice keeps its original translational symmetry. Therefore, the supercell of our distorted heterostructure must have the following dimensions: $T$ along the $x$-axis; $t$ along the $z$-axis and a unit cell along the $y$-axis. Because $T$ and $t$ are equal to several times the unit cell dimension, we need to realize a supercell containing thousands of atoms. In order to do this we implemented a computer program with the software MATHEMATICA [10], which allows us to calculate the non-uniform strain fields generated by the elastic relaxation of the compositional stresses in thinned superlattices and to realize the extended supercelles which are necessary to describe the atomic configuration of the deformed lattice in a volume equal to $T t d$. In particular, the implemented program is compatible with the simulation package CERIUS-HRTEM [11]. All the parameters of the supercell (type of atom, atomic coordinates, and so on) are given in the format required by this simulation program. By using the strained semiconductor superlattice model generated by means of the MATHEMATICA software in the CERIUS-HRTEM simulation program we can study the influence of the elastic relaxation on the HRTEM image contrast.

In order to verify this influence we created a supercell from silicon with the same dimensions of the superlattice shown in Figure 2 and with the same lattice distortion. The only difference is 


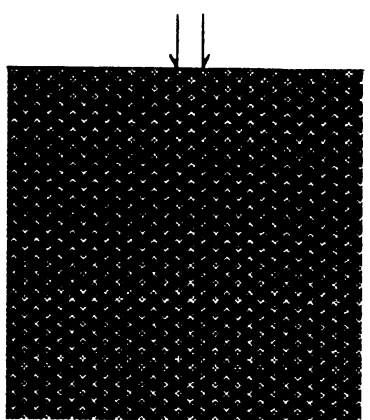

$\operatorname{def}=-72.75 \mathrm{~nm}$

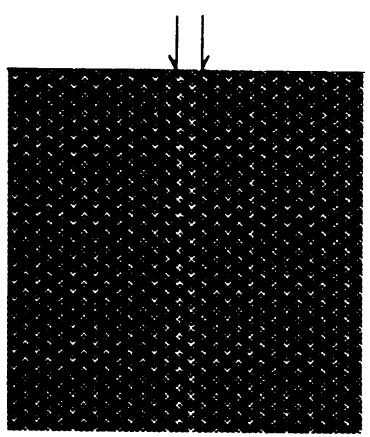

$\operatorname{def}=-42.12 \mathrm{~nm}$

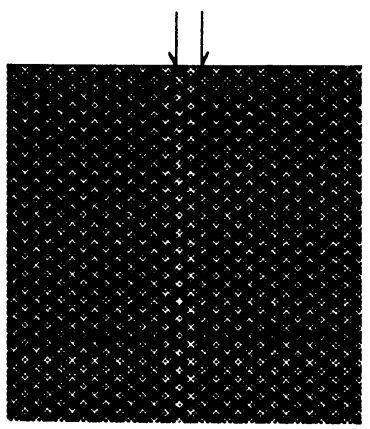

$\operatorname{def}=-11.50 \mathrm{~nm}$

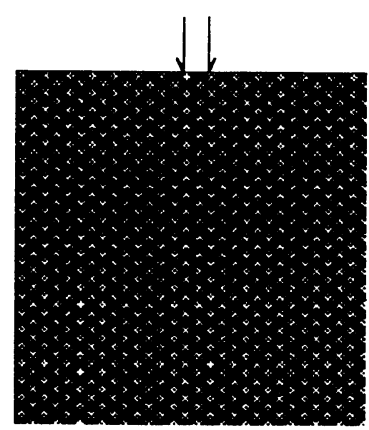

$\operatorname{def}=-62.54 \mathrm{~nm}$

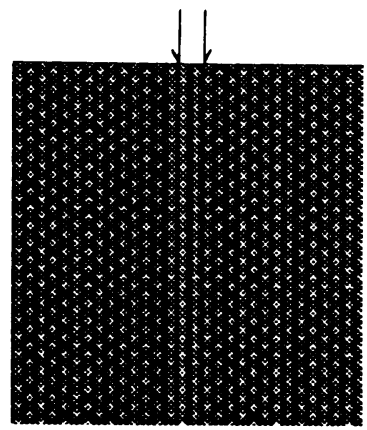

$\operatorname{def}=-31.91 \mathrm{~nm}$

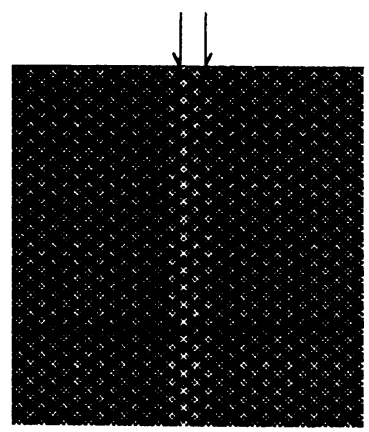

$\operatorname{def}=-1.29 \mathrm{~nm}$

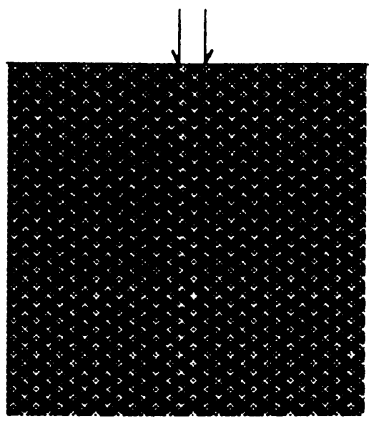

$\operatorname{def}=-52.33 \mathrm{~nm}$

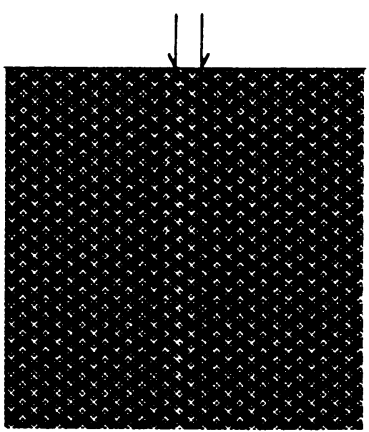

$\operatorname{def}=-21.71 \mathrm{~nm}$

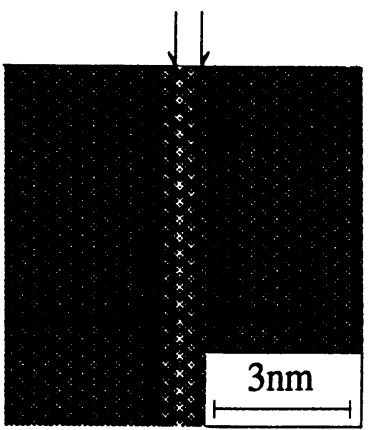

$\operatorname{def}=8.92 \mathrm{~nm}$

Fig. 3. - Simulated through-focus HRTEM images in a $<011>$ zone axis for a crystal of silicon with the same dimensions of the superlattice shown in Figure 2 and with the same lattice distortion. The only difference is that the germanium atoms present in the superlattice have been substituted with silicon atoms (see text). The arrows indicate the position of the interfaces. The marker is equal for all the images.

that the germanium atoms present in the superlattice have been substituted with silicon atoms. In this way, we have eliminated the chemical contribution to the HRTEM contrast due to different atomic species. Figure 3 shows a simulated through-focus HRTEM images in a $<011>$ zone axis which demonstrates how the lattice deformation contributes to the HRTEM image contrast, for some defocus values. We used several thickness values for the simulation ranging from 5 to $20 \mathrm{~nm}$. The presence of the elastic relaxation is always detectable, even if it is more evident for some thickness values. The electro-optical parameters used for this simulation are: accelerating 


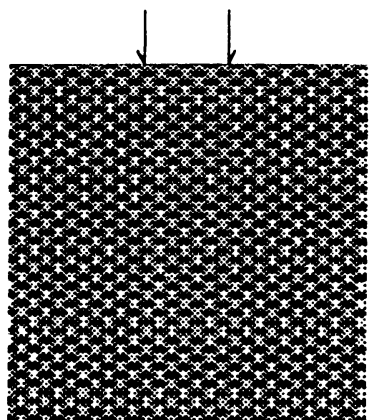

def $=-77.77 \mathrm{~nm}$

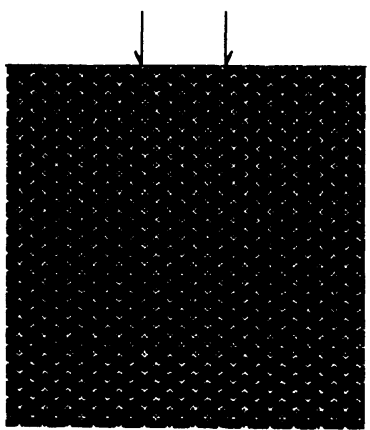

$\operatorname{def}=-44.44 \mathrm{~nm}$

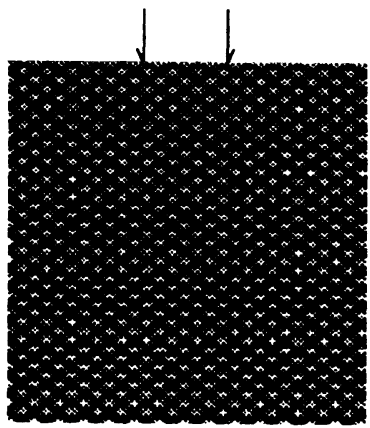

a)

$\operatorname{def}=-11.11 \mathrm{~nm}$

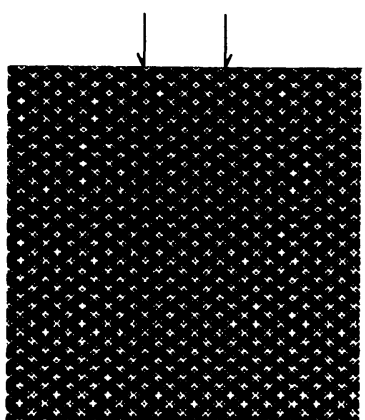

$\operatorname{def}=-66.66 \mathrm{~nm}$

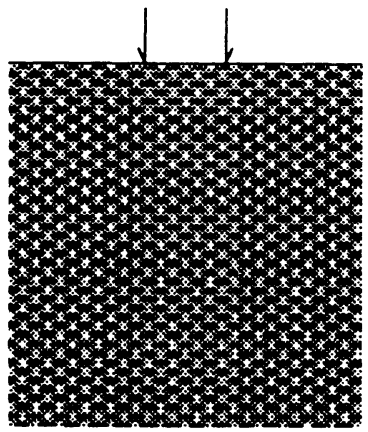

$\operatorname{def}=-33.33 \mathrm{~nm}$

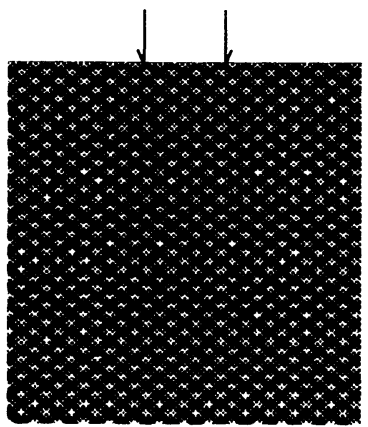

$\operatorname{def}=0 \mathrm{~nm}$

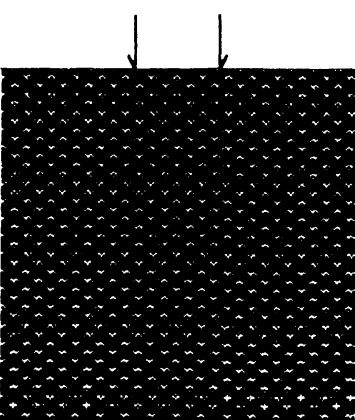

$\operatorname{def}=-55.55 \mathrm{~nm}$

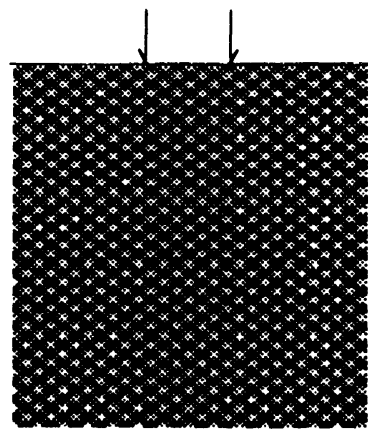

$\operatorname{def}=-22.22 \mathrm{~nm}$

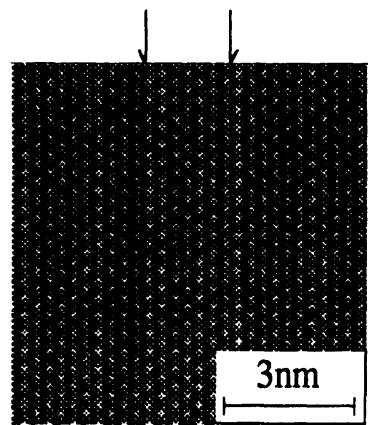

$\operatorname{def}=-41.55 \mathrm{~nm}$

Fig. 4. - a) Simulated through-focus HRTEM images in a $<011>$ zone axis for a $\mathrm{GaAs} / \mathrm{In}_{0.095} \mathrm{Ga}_{0.905} \mathrm{As}$ superlattice realized by means of the package CERIUS-INTERFACE. The arrows indicate the position of the interfaces. The marker is equal for all the images. b) Simulated through-focus HRTEM images in a $<011>$ zone axis for a $\mathrm{GaAs} / \mathrm{In}_{0.095} \mathrm{Ga}_{0.905}$ As superlattice realized by means of our model, which considers the lattice bending. The arrows indicate the position of the interfaces. The marker is equal for all the images.

voltage $300 \mathrm{kV}$, spherical aberration coefficient $2 \mathrm{~mm}$, objective diaphragm radius $4 \mathrm{~nm}^{-1}$, beamdivergence semi-angle $1 \mathrm{mrad}$, Scherzer defocus $-62.75 \mathrm{~nm}$.

A further example of a material system widely characterized by HRTEM is reported in Figure 4 which shows a direct comparison between the HRTEM simulated images in a $<011>$ zone axis, for a $\mathrm{GaAs} / \mathrm{In}_{0.095} \mathrm{Ga}_{0.905}$ As superlattice, obtained directly by the CERIUS program, i.e. without elastic lattice relaxation (Fig. 4a), and by using our model (Fig. 4b). We have put $a=9.05 \mathrm{~nm}$, 


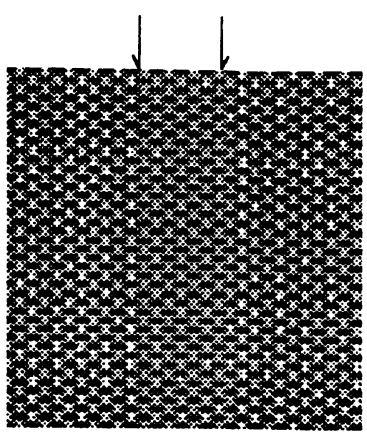

$\operatorname{def}=-77.77 \mathrm{~nm}$

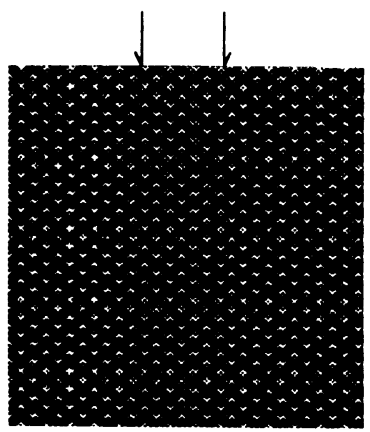

$\operatorname{def}=-44.44 \mathrm{~nm}$

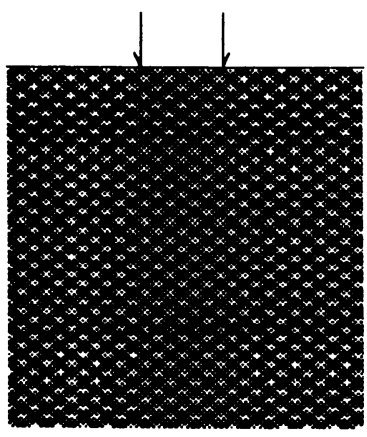

$\operatorname{def}=-11.11 \mathrm{~nm}$

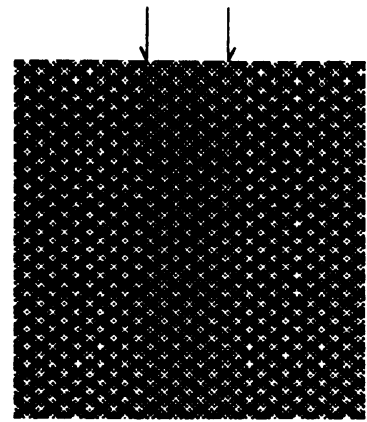

$\operatorname{def}=-66.66 \mathrm{~nm}$

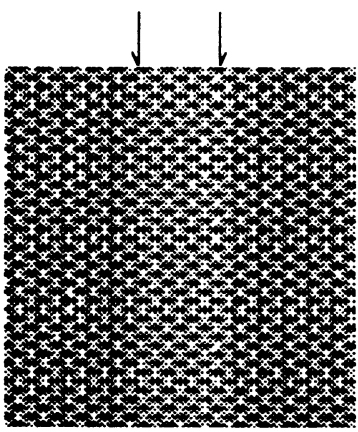

$\operatorname{def}=-33.33 \mathrm{~nm}$

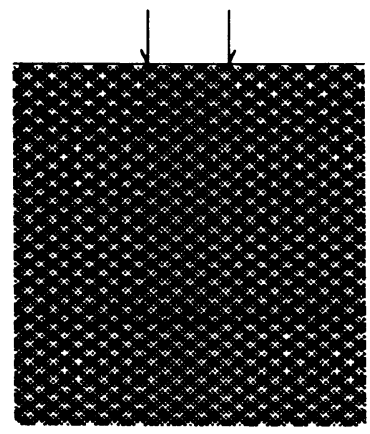

$\operatorname{def}=0 \mathrm{~nm}$

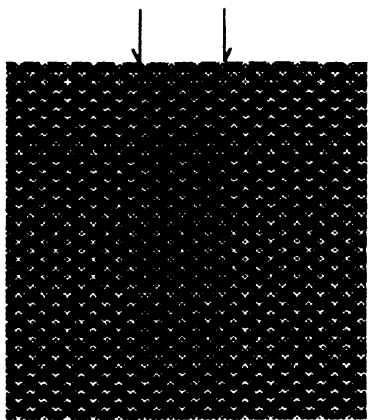

$\operatorname{def}=-55.55 \mathrm{~nm}$

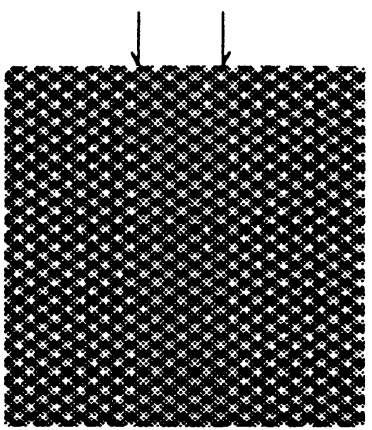

$\operatorname{def}=-22.22 \mathrm{~nm}$

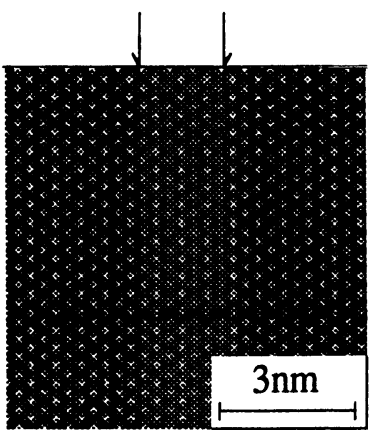

$\operatorname{def}=-41.55 \mathrm{~nm}$

b)

Fig. 4. - (Continued.)

$b=2.28 \mathrm{~nm}$ and $t=8.5 \mathrm{~nm}$, where $a$ and $b$ are, respectively, the widths of materials A and B, and $t$ the specimen thickness. The values of the electro-optical parameters are: accelerating voltage $400 \mathrm{kV}$, spherical aberration coefficient $1.05 \mathrm{~mm}$, objective diaphragm radius $5.4 \mathrm{~nm}^{-1}$, beamdivergence semi-angle $0.80 \mathrm{mrad}$, Scherzer defocus $-41.55 \mathrm{~nm}$. It is interesting to note that the CERIUS simulation program, which does not consider the elastic lattice relaxation in the building process of the heterointerfaces, gives a negligible contrast for all the considered defocus values. On the contrary, for some defocus values (even at the Scherzer value), our model enhances the presence of the heterostructure, due to the lattice bending. 


\section{Conclusions}

Our results show that: i) elastic relaxation gives a contribution to the contrast variation of HRTEM images of strained semiconductor superlattices; ii) this contribution has to be taken into account whenever HRTEM is used to deduce the local chemical composition or the local unit cell dimensions in strained semiconductor materials; iii) these lattice distortions could be used for enhancing the image contrast when the chemical contrast, caused by the different scattering factors of different atomic species, is negligible.

\section{References}

[1] Brown J.M., Holonyak Jr H., Kaliski R.W., Ludowise M.J., Dietze W.T. and Lewis C.R., Appl. Phys. Lett. 44 (1984) 1158.

[2] Kobayashi M., Konagai M., Takahashi K. and Urabe K., J. Appl. Phys. 61 (1987) 1015.

[3] Gibson J.M. and Treacy M.M.J., Ultramicrosc. 14 (1984) 345.

[4] Treacy M.M.J. and Gibson J.M., J. Vac. Sci. Technol. B4 (1986) 1458.

[5] Treacy M.M.J., Gibson J.M. and Howie A., Philos. Mag. A51 (1985) 389.

[6] Perovic D.D., Weatherly G.C. and Houghton D.C., J. Vac. Sci. Technol. A6 (1988) 1333; Philos. Mag. A64 (1991) 1.

[7] Bierwolf R., Hohenstein M., Phillipp F., Brandt O., Crook G.E. and Ploog K., Ultramicrosc. 49 (1993) 273.

[8] Giuffrida A., Thesis D., Università di Lecce (1995).

[9] De Caro L., Giuffrida A., Carlino E. and Tapfer L., in progress.

[10] MATHEMATICA Software, release 2.2, Wolfram Research Inc., Champaign, Illinois U.S.A.

[11] CERIUS Molecular Modeling Software for Materials Research from Molecular Simulation Inc., Cambridge UK.

[12] Amendaze Yu. A., “Theory of Elasticity” (Mir Publishers, Moscow, 1979). 